\title{
Rol del factor VII recombinante activado en pacientes neuroquirúrgicos y neu- rocríticos
}

\author{
P. Rama-Maceiras; I. Ingelmo-Ingelmo*; N. Fàbregas-Julià** y J. Hernández-Palazón***
}

Servicio de Anestesiología y Reanimación. Complejo Hospitalario Universitario A Coruña. *Hospital Universitario Ramón y Cajal. Madrid. Hospital Clínic Barcelona. Universitat de Barcelona. ${ }^{* * *}$ Hospital Universitario Virgen de la Arrixaca. Murcia.

Resumen

La hemorragia del sistema nervioso central constituye una patología grave, ya que un volumen de sangrado relativamente pequeño en el cerebro puede presentar consecuencias devastadoras. La utilización de agentes hemostáticos pueden reducir las consecuencias de la hemorragia cerebral espontánea, traumática o secundaria a la anticoagulación. El Factor VII recombinante activado (rFVIIa) es un fármaco procoagulante que se ha empleado en diversas patologías hemorrágicas del sistema nervioso central, si bien esta indicación no se recoge en la ficha técnica del fármaco. En esta revisión se repasa el mecanismo de actuación del fármaco, su potencial en el tratamiento de la hemorragia del sistema nervioso central y las evidencias existentes al respecto.

Se realizó una revisión de la bibliografía disponible sobre el uso de rFVIIa en el control de la hemorragia relacionada con el sistema nervioso central, la patología neurocrítica y procedimientos neuroquirúrgicos, mediante búsqueda en MEDLINE y en el registro de ensayos clínicos: $\mathrm{http}$ ://clinicaltrials.gov (última revisión septiembre 2010), además de revisión manual de las publicaciones a partir de la bibliografía de los artículos recuperados.

En la hemorragia cerebral espontánea, existen ensayos clínicos aleatorios, cuyos resultados desaconsejan la utilización sistemática del fármaco en esta indicación. En el resto de hemorragias relacionadas con el sistema nervioso central, los datos existentes se basan en estudios retrospectivos, opiniones de expertos o casos clínicos aislados.

PALABRAS CLAVE. Desórdenes de la coagulación. Hemorragia intracraneal. Factor VII recombinante activado. Cuidados críticos.

The role of recombinant activated factor VII in neurosurgical and neurocritical patients

Recibido: 20-06-10. Aceptado: 18-10-10
Summary

Central nervous system haemorrhage is a severe pathology, as a small amount of bleeding inside the brain can result in devastating consequences. Haemostatic agents might decrease the consequences of intracranial bleeding, whichever spontaneous, traumatic, or anticoagulation treatment etiology. Proacogulant recombinant activated factor VII (rFVIIa) has been given after central nervous system bleeding, with an off-label indication. In this update, we go over the drug mechanism of action, its role in the treatment of central nervous system haemorrhage and the published evidences regarding this subject.

We carried out a literature review concerning the treatment with rFVIIa in central nervous system haemorrhage, neurocritical pathologies and neurosurgical procedures, searching in MEDLINE and in clinical trials registry: http://clinicaltrials.gov (last review September 2010), as well as performing a manual analysis of collected articles, looking for aditional references.

The results of randomized clinical trials do not support the systematic administration of rFVIIa for spontaneous intracranial cerebral haemorrhage. In other central nervous system related haemorrhages, the current available data consist on retrospective studies, expert opinion or isolated case reports.

KEY WORDS. Blood coagulation disorders. Intracranial haemorrhages. Recombinant FVIIa. Critical care.

\section{Introducción}

La enfermedad cerebrovascular aguda (ictus, apoplejía, stroke), es una neuropatología grave con alto riesgo de mortalidad o discapacidad grave ${ }^{27,36}$. La Organización Mundial de la Salud estima que un stroke sucede cada cinco segundos y constituye de forma global la segunda causa de mortalidad ${ }^{16}$. A pesar de las dificultades en evaluar los gastos indirectos, su costo total se estima en 65,5 billones de dolares ${ }^{48,68}$. 
El accidente cerebrovascular se clasifica en hemorrágico e isquémico ${ }^{6}$, siendo la forma hemorrágica menos frecuente que la isquémica (13\% vs $87 \%$ ). El accidente hemorrágico incluye la hemorragia (hematoma) intracerebral (HIC), la subaracnoidea (HSA) y la intraventricular (HIV), con mayor prevalencia de la HIC sobre la HSA $(10 \% \text { vs } 3 \%)^{1,48}$. La ubicación del hematoma, por orden de mayor a menor frecuencia, es la siguiente: profunda, lobar, cerebelosa y tronco cerebral $^{42}$. La gravedad de las hemorragias viene marcada porque a nivel del sistema nervioso central (SNC), un volumen de sangrado relativamente pequeño puede presentar consecuencias devastadoras. Los agentes hemostáticos podrían reducir estas consecuencias. El factor VII recombinante activado (aFVIIr) es un fármaco procoagulante que se ha empleado en diversas patologías hemorrágicas del SNC; si bien esta indicación no se recoge en la ficha técnica del fármaco.

El objetivo de esta revisión es repasar el potencial terapéutico del aFVIIr en la hemorragia del SNC y las evidencias existentes sobre seguridad y eficacia al respecto. Para ello se realizó una búsqueda en MEDLINE (última revisión en septiembre 2010), usando los Medical Subject Headings ("Intracranial hemorrhages" o "Neurosurgery" o "Neurosurgical procedures") y "recombinant Factor VIIa" (Substance name) o "Factor VIIa", sin restricciones en cuanto a idioma o tipo de publicación. Asimismo se realizó una búsqueda en el registro de ensayos clínicos: http: //clinicaltrials.gov. Además, en la bibliografía de los artículos recuperados se buscaron nuevas referencias relevantes. Se obtuvieron 107 publicaciones referidas al uso del aFVIIr en la hemorragia relacionada con el SNC, procedimientos neuroquirúrgicos y pacientes neurocríticos.

\section{El nuevo modelo de coagulación. El factor VII}

La disrupción vascular y/o lesión tisular ponen en marcha una serie de mecanismos de hemostasia que comprenden la vasoconstricción local, el depósito y agregación de plaquetas, y la coagulación de la sangre. La coagulación es el proceso enzimático por el cual el fibrinógeno soluble se convierte en fibrina insoluble, capaz de polimerizar ${ }^{49}$. Los antiguos conceptos in vitro de hemostasia ${ }^{32,84,99}$ se han suplantado por un modelo más fisiológico (celular) que ocurre in vivo, donde la coagulación es un fenómeno enzimático en el que intervienen tanto componentes de la sangre como de los tejidos. La nueva cascada, incluye 3 fases: la inicial, la de amplificación y la de propagación (Figura 1). Esta visión hace énfasis en el papel de las células como fuente de factor tisular (FT) y fosfolípidos de superficie, para integrar los factores de la coagulación ${ }^{61}$.

El nivel de actividad coagulante en el humano es en condiciones basales muy bajo ${ }^{85}$ y se mantiene mediante la asociación de pequeñas cantidades de complejo FT/FVII a nivel extravascular ${ }^{57}$. En determinadas situaciones, como tras una lesión cerebral aguda, se produce una liberación masiva de FT y otras tromboplastinas desde el tejido dañado, que hiperactivan la coagulación y la fibrinolisis ${ }^{10}$, por lo que pueden desarrollarse discoagulopatías que incrementan la hemorragia $44,57,109,118,149$. Dicha coagulopatía se agrava con la hiperfibrinolisis, el consumo de factores y la anemia ${ }^{10,57,88}$. La hipoxia, la hipoperfusión y la liberación de catecolaminas y esteroides en respuesta a la lesión, también pueden favorecer la coagulopatía asociada a las lesiones cerebrales ${ }^{97,100}$. Además, la trombina liberada puede producir vasoconstricción, reducción del flujo sanguíneo cerebral y activación de proteinasas, que dañan la barrera hematoencefálica y favorecen el edema cerebral ${ }^{118}$.

El eptacog alfa o aFVIIr (NovoSeven $\AA$, Novo Nordisk, Bagsvaerd, Dinamarca), es un análogo de la sustancia endógena de $50 \mathrm{kDa}^{70}$, sintetizado por ingeniería genética y libre de proteínas humanas ${ }^{33}$ que puede potenciar la hemostasia en procesos hemorrágicos que no responden a las terapias convencionales ${ }^{115}$. La concentración alcanzada con una dosis de $90 \mu \mathrm{g} \bullet \mathrm{Kg}^{-1}$ representa más de 300 veces los niveles circulantes normales del FVII ${ }^{56,115}$, sin que se produzca una activación de la coagulación generalizada, por lo que se cree que su mecanismo de acción es local. Por un lado debido a la unión con el FT, activa la coagulación a nivel de la lesión endotelial. Por otro, dosis elevadas del fármaco actúan al unirse a las plaquetas activadas a nivel de la lesión, activando el FX independientemente del papel del $\mathrm{FT}^{34}$. La formación de trombina inhibe la fibrinolisis, reforzando el coágulo de fibrina ${ }^{57,77}$. In vitro, el fármaco parece acortar el tiempo requerido para alcanzar la velocidad máxima de formación del coágulo ${ }^{122}$. Tras la administración del preparado, el inicio de acción es inmediato y la respuesta clínica puede observarse en 10 minutos.

La principal indicación aprobada del aFVIIr es la hemofilia con aloanticuerpos (inhibidores) para los factores VIII y $\mathrm{IX}^{57,77,127}$. La actividad del fármaco es menor que la del FVIII o el IX en la hemofilia A o B sin inhibidores, para las que también se ha aprobado su uso ${ }^{115}$; así como para el déficit congénito de factor $\mathrm{VII}^{67,146}$. Además en Europa es apto para la tromboastenia de Glanzmann resistente a la transfusión de plaquetas $^{57,75}$. La mayoría del conocimiento disponible sobre el mecanismo de acción del fármaco se basa en su uso en la hemofilia, pero su comportamiento no tiene por qué ser idéntico en otras situaciones ${ }^{18,70}$ y se cree que la producción de trombina es superior en los pacientes no hemofílicos ${ }^{106}$.

A pesar de que no existen evidencias que apoyen definitivamente su uso ${ }^{124}$, la eficacia del fármaco ha extendido su aplicación a otras situaciones que se incluyen como fuera de guía clínica o de uso compasivo (off-label $)^{5}$, por ejemplo: en el déficit congénito de factor $\mathrm{XI}^{133}$, trombocitopenias refractarias $^{142}$ y trombopatías como la púrpura trombocitopénica, así como en el síndrome de Bernard Soulier o 


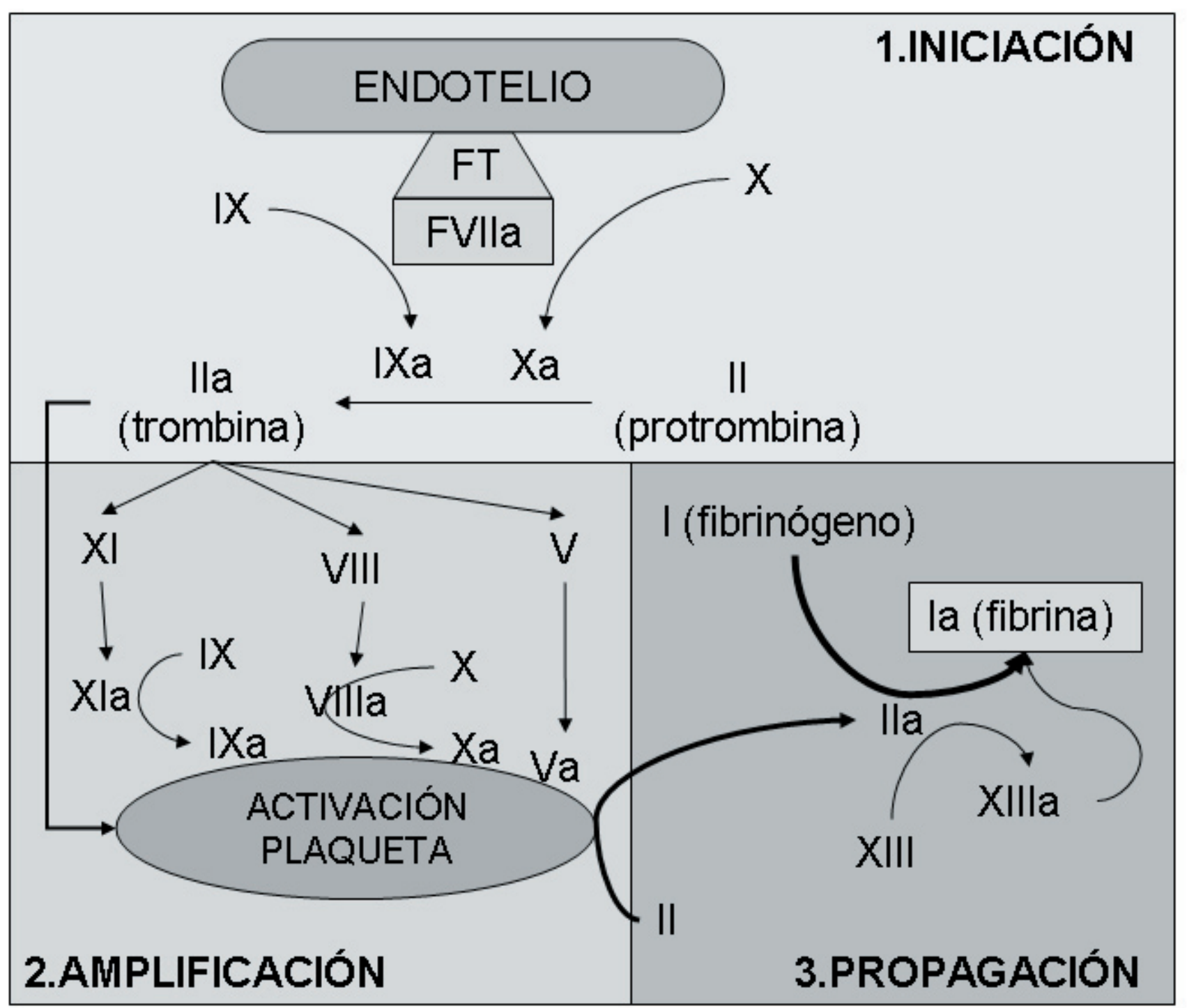

Figuta 1. Esquema del modelo fisiológico (celular) de la coagulación (adaptado de ${ }^{53,57,112}$ ).

FT: Factor tisular. FVII: Factor VII. IX: Factor IX (Christmas o antihemofilico B). X: Factor X (Stuart-Prower). XI: Factor XI. V: Factor V (Proacelerina). VIII: Factor VIII (antihemofilico A). XIII: Factor XIII. El subíndice " $a$ " indica en todos los casos que el factor está activado.

En la fase de iniciación, el complejo FVII-FT, expuesto en las zonas de disrupción endotelial, provoca la activación de los factores IX y X y la formación de pequeñas cantidades de trombina. Esta trombina participa en la fase de amplificación al colaborar en la activación de la plaqueta, en cuya superficie se acumulan gran cantidad de factores activados. En la fase de propagación, esos factores generan grandes cantidades de trombina y esta a su vez al fibrina, posteriormente estabilizada por el factor XIII.

la enfermedad de von Willebrand ${ }^{77,115}$ y en la hemorragia postparto o complicaciones obstétricas. Otras aplicaciones relevantes son: a) la hemorragia gastrointestinal, la asociada a pancreatitis o por quemadura extensa, b) en los traumatismos graves y/o hemorragias con riesgo vital cuando fracasan las medidas convencionales ${ }^{11,86,115}, \mathrm{y}$ c) en pacientes con déficit de producción de trombina ${ }^{76}$. También parece presentar eficacia en el bloqueo de los efectos de nuevos fármacos anticoagulantes como el fondaparinux, del que no dispone de antídoto específico ${ }^{12,77,96}$ y el idraparinux, un pentasacárido con una vida media de 5,5 días $^{13}$.

La eficacia del aFVIIr puede disminuir cuando hay déficit de otros factores ${ }^{122}$, pero especialmente en presencia de la triada acidosis-hipotermia-hemodilución ${ }^{53}$. En caso 
Tabla 1

Resumen de las series retrospectivas publicadas sobre la indicación "off-label" del aFVIIr en pacientes neurocríticos y neurocirugía

\begin{tabular}{|c|c|c|c|c|c|}
\hline Indicación & $\begin{array}{l}\text { HIC asociada a ACO } \\
\text { o coagulopatía } 8,19,20, \\
24,26,31,43,62,63,65,80,81,94, \\
98,103,117,118,122,135,142\end{array}$ & $\begin{array}{l}\text { TCE con o sin } \\
\text { coagulopatía } 9,17, \\
22,72,97,100,118,125,126, \\
141,144,148,149\end{array}$ & $\mathrm{HSA}^{34,111,120}$ & $\underset{46,54,59,60,66,131,133,139}{\text { Qx programada }}$ & $\begin{array}{c}\text { Mixtos } \\
30,40,108,130\end{array}$ \\
\hline $\mathrm{N}^{0}$ de pacientes & 245 & 183 & 29 & 23 & 118 \\
\hline Dosis $\left(\mu \mathrm{g} \bullet \mathrm{kg}^{-1}\right)$ & $4,4-202$ & $6-200$ & $40-160$ & $20-220$ & $23-270$ \\
\hline PFC & $59 / 90(65,5 \%)$ & $36 / 56(64,2 \%)$ & No com & $11 / 15(73,3 \%)$ & $96 / 118(81,3 \%)$ \\
\hline Vitamina $\mathrm{K}$ & $153 / 183(83,6 \%)$ & $11 / 19(57,9 \%)$ & No com & No com & $56 / 93(60,2 \%)$ \\
\hline Comp. Tromb. & $23 / 235(9,8 \%)$ & $9 / 73(12,3 \%)$ & $2 / 29(6,8 \%)$ & $0(0 \%)$ & $6 / 118(5,0 \%)$ \\
\hline Mortalidad & $57 / 206(27,6 \%)$ & $29 / 70(41,4 \%)$ & $5 / 19(26,3 \%)$ & $1 / 15(6,6 \%)$ & $40 / 109(36,7 \%)$ \\
\hline Discapacidad grave & $13 / 46(28,2 \%)$ & $4 / 15(26 \%)$ & No com & $1 / 13(7,6 \%)$ & No com \\
\hline Buena recuperación & $20 / 45(44,4 \%)$ & $4 / 8(50 \%)$ & No com & $13 / 15(86,6 \%)$ & $10 / 93(10,7 \%)$ \\
\hline
\end{tabular}

HIC: hemorragia intracerebral. PFC: plasma fresco congelado. ACO: anticoagulantes orales. TCE: traumatismo craneoencefálico. HSA: hemorragia subaracnoidea. Qx: cirugía. Comp. Tromb.: complicaciones trombóticas. No com: No se comunica en las series. Los denominadores de los diferentes apartados muestran el número de pacientes en los que se ha podido recoger la variable indicada.

de acidosis importante $(\mathrm{pH}<7,1)$, hasta un $90 \%$ del aFVIIr puede inactivarse ${ }^{10}$. También parece menos eficaz cuando el paciente presenta lesión a nivel de varias cavidades corporales y en los casos graves con shock hemorrágico profundo ${ }^{21}$. La respuesta parece ser más efectiva en presencia de un recuento plaquetario adecuado, aunque se requiera transfusión de las mismas ${ }^{127}$.

Pese a su efecto predominante local, el principal riesgo del aFVIIr es la aparición de fenómenos trombóticos, especialmente arteriales, cuando contacta con placas ateroescleróticas; particularmente del cerebro y corazón $^{34,35,107}$. Sin embargo, las trombosis venosas no parecen ser frecuentes ya que dependen más de un mecanismo de estasis ${ }^{34}$. Por otro lado, el fármaco puede favorecer la liberación de citoquinas proinflamatorias ${ }^{87}$. Los eventos trombóticos se han situado en torno al 1-2\% en los pacientes hemofílicos ${ }^{77,85}$, aunque la incidencia parece mayor en las indicaciones "off-label" $53,86,103,106,116$ y con dosis elevadas del fármaco ${ }^{86}$. Una revisión de 13 ensayos clínicos realizados hasta 2005 , en indicaciones ajenas a la hemofilia, mostró un $5,3 \%$ de eventos adversos en los pacientes que recibían placebo, frente a un $6,0 \%$ en los que recibían el tratamiento activo ${ }^{78}$. Una revisión sistemática de la literatura de 2007 sólo demostró una tendencia, no significativa, al aumento de las complicaciones trombóticas en los pacientes incluidos en ensayos clínicos realizados en las indicaciones fuera de guía clínica ${ }^{124}$. Muchas de las complicaciones comunicadas se produjeron en ancianos con diabetes, ateroesclerosis u obesidad (situaciones que pueden promover la hipercoagulabilidad $)^{56,77,115}$, o en pacientes a los que se les administró el aFVIIr asociado a otros fármacos procoagulantes, por ejemplo el concentrado de complejo protrombínico $(\mathrm{CCP})^{56}$. El empleo de antifibrinolíticos asociado al aFVIIr es discutible, porque si bien ambos son protrombóticos, los antifibrinolíticos promueven la inhibición del $\mathrm{FT}^{56}$.

Contraindicaciones absolutas para el empleo del aFVIIr son la hipersensibilidad al fármaco o alguno de sus componentes (proteínas del ratón, hámster y bovinas) ${ }^{41} \mathrm{y}$ los pacientes considerados no recuperables por consenso médico ${ }^{88}$. La lesión vascular mayor tampoco es una indicación adecuada ${ }^{115}$. Las contraindicaciones relativas incluyen una historia de eventos tromboembólicos en los 6 meses previos, pacientes con riesgo trombótico elevado, o con enfermedad ateroesclerótica conocida; especialmente si la placa es inestable, porque hay más exposición de $\mathrm{FT}^{77,88}$. La coagulación intravascular diseminada (CID) secundaria a la sepsis, es otra situación de riesgo de activación generalizada de la coagulación al emplear el aFVIIr, por la exposición del FT por los monocitos ${ }^{56,77}$. 


\section{Empleo del aFVIIr en patología neurocrítica}

En neuropatología quirúrgica y/o neurointensivismo, el control precoz de la hemorragia es importante ${ }^{45}$, pues pequeños volúmenes pueden tener consecuencias devastadoras ${ }^{56}$ y la politransfusión incrementa el riesgo de edema cerebral ${ }^{65}$. Pese a que el número de publicaciones de series de casos relativas al empleo de aFVIIr en pacientes neurocríticos se incrementa día a día (Tabla 1), los estudios disponibles hasta el momento no apoyan el uso del aFVIIr en ninguna indicación concreta. Los ensayos clínicos que muestran beneficios son retrospectivos y presentan lagunas metodológicas importantes, además del posible sesgo de comunicación de efectos secundarios; mientras que los ensayos prospectivos no han mostrado beneficio del fármaco. Actualmente, la indicación en patologías neuroquirúrgicas o neurocríticas es "off-label"; no obstante, su utilización se ha incrementado como adyuvante de la hemostasia en situaciones puntuales, como la HIC secundaria a anticoagulación (acenocumarol, warfarina) y en la hemorragia masiva perioperatoria o traumática que se muestra refractaria al tratamiento habitual. Por hemorragia masiva entendemos la necesidad de más de 8 unidades de concentrado de hematíes durante las primeras 12 horas de tratamiento o más de 10 en las primeras $24^{10}$.

Una revisión realizada en 2008 de la base de datos haemostasis.com, de comunicación libre del empleo del aFVIIr, mostró 16 pacientes neurocríticos con patología diversa (la mayoría neurotraumas), tratados con dosis de 31-270 $\mu \mathrm{g} \mathrm{Kg}^{-1}$, para la estabilización del sangrado intracraneal, el control del sangrado perioperatorio y/o la corrección de la coagulopatía. La respuesta hemostática fue eficaz en 14 de los 16 casos. Cuatro pacientes fallecieron; uno por sangrado incontrolable, otro por edema cerebral en el primer día de evolución y 2 por fracaso multiorgánico más tardío. Se produjo un caso de elevación masiva de los dímeros $\mathrm{D}$, que se relacionó con la administración del fár$\operatorname{maco}^{40}$.

A continuación se repasan las evidencias relativas al empleo del aFVIIr en las principales patologías neurocríticas:

\section{a. Hemorragia intracerebral espontánea o primaria}

Constituye entre el $10-30 \%$ de todos los ictus y el tipo más serio ${ }^{89}$. Suele atribuirse a rotura de pequeñas arterias cerebrales penetrantes ${ }^{58}$, aunque la hipertensión, los microaneurismas de Charcot-Bouchard y la angiopatía amiloide son factores etiológicos y/o precipitantes clási$\cos ^{89,143}$.

Existe incertidumbre sobre la efectividad de la neurocirugía en estos procesos, por lo que las medidas de soporte vital y los tratamientos médicos como el control de la presión arterial y los fármacos procoagulantes podrían jugar un papel importante en su terapia ${ }^{7,89,93,128}$.

Los resultados de los ensayos clínicos en fase II del empleo de aFVIIr en estos pacientes fueron prometedores en cuanto a eficacia (reducción del tamaño del hematoma y de la mortalidad) ${ }^{91,93}$, en cuanto a seguridad (eventos tromboembólicas del $8 \%$ con el tratamiento frente a $5 \%$ con placebo $)^{34} \mathrm{y}$ en el análisis coste-beneficio ${ }^{71}$. Sin embargo, los hallazgos del ensayo en fase III (terapia hemostática ultratemprana), que comparaba el tratamiento con dosis de 20 ó $80 \mu \mathrm{g} \cdot \mathrm{Kg}^{-1}$ de aFVIIr frente a placebo en las 4 primeras horas de evolución de la HIC no asociada a anticoagulación previa, mostraron ausencia de efecto sobre la mortalidad o la discapacidad grave con el tratamiento ${ }^{89,92}$, pese a que se mantuvo la reducción del tamaño del hematoma observado en los ensayos iniciales ${ }^{138}$. Además, la prevalencia de los fenómenos trombóticos arteriales fue superior en el grupo de pacientes tratados con $80 \mu \mathrm{g} \cdot \mathrm{Kg}^{-1}$ respecto al placebo ${ }^{92}$. Este aumento se produjo especialmente a expensas de los síndromes coronarios agudos sin elevación del segmento ST o episodios aislados de elevación de troponinas ${ }^{35}$. La repercusión clínica de estos cuadros es dudosa y además podría relacionarse con la patología neurológica, al igual que ocurre en la HSA ${ }^{34,134}$.

La presencia de accidentes trombóticos arteriales se relacionó con una dosis elevada del fármaco $(80$ $\mu \mathrm{g} \cdot \mathrm{Kg}^{-1}$ ), la presencia previa de signos de isquemia cerebral o miocárdica, la edad avanzada y el uso previo de antiagregantes plaquetarios ${ }^{35}$. No hubo diferencias en los eventos venosos (trombosis venosa profunda o embolismo pulmonar), ni en el desarrollo de edema cerebral que se podría esperar por un exceso en la formación de trombina $^{93}$.

El citado ensayo en fase III $^{92}$, criticado por los criterios de inclusión empleados ${ }^{71}$ ha frenado la utilización del aFVIIr en la HIC, aunque sugiere un posible efecto beneficioso en pacientes con riesgo elevado de expansión del hematoma ${ }^{135,143}$ o en algunos subgrupos concretos: $<70$ años, volumen de hematoma $\leq 60 \mathrm{~mL}$, extensión ventricular $\leq 5 \mathrm{~mL}$, y tiempo desde el inicio de los síntomas hasta el tratamiento de $\leq 2,5$ horas $^{86,105}$; aunque como todo análisis de subgrupos, estos hallazgos deberían servir para generación de nuevas hipótesis de investigación, más que como resultado definitivo ${ }^{47}$.

Otras series no aleatorizadas de pacientes con HIC que se trataron con aFVIIr, insisten en el aumento de complicaciones trombóticas, elevación de troponinas e infarto de miocardio ${ }^{134}$, así como en el empeoramiento de la hidrocefalia en caso de extensión intraventricular del sangrado ${ }^{132}$. Estas series han sido criticadas por el escaso tamaño muestral y posibles sesgos en el análisis de los pacientes y los datos $^{28,29,90}$.

Las recientes guías de la American Heart Association (AHA) sobre el tratamiento de la HIC espontánea conclu- 
yen que aunque el aFVIIr puede limitar la extensión del hematoma, hay un aumento de riesgo tromboembólico sin un beneficio claro en pacientes no seleccionados; por lo que la administración del aFVIIr no se recomienda de forma no selectiva (recomendación clase III, nivel de evidencia A). Se necesitan nuevos estudios para determinar si algún subgrupo de pacientes podría beneficiarse de este tratamiento ${ }^{50,101}$.

La hemorragia intraventricular (HIV) se asocia frecuentemente a la HIC o a la HSA y constituye un factor pronóstico negativo ${ }^{64}$. Se ha demostrado una reducción de la progresión de la HIV en $24 \mathrm{~h}$ tras el tratamiento con aFVIIr $^{129}$. Incluso se ha combinado el fármaco para control de la hemorragia con la trombolisis intratecal, para evitar la obstrucción de los drenajes ventriculares por coágulos ${ }^{64}$.

Aunque la población y la etiopatogenia del problema sean diferentes ${ }^{116}$, se ha sugerido el papel profiláctico del aFVIIr en la HIV del neonato prematuro ${ }^{39,140}$; aunque se necesitan ensayos clínicos que confirmen su eficacia y la ausencia de complicaciones ${ }^{51,95}$.

\section{b. Hemorragia subaracnoidea espontánea y procedimien- tos neurovasculares}

La prevención del resangrado tras rotura de aneurisma intracraneal es fundamental. Un ensayo clínico sobre seguridad y dosificación del aFVIIr se suspendió cuando uno de los diez pacientes, tratado con $80 \mu \mathrm{g} \bullet \mathrm{Kg}^{-1}$ en bolo y perfusión de $7 \mu \mathrm{g} \bullet \mathrm{Kg}^{-1} \cdot \mathrm{h}^{-1}$, presentó al $4^{\circ}$ día de evolución un cuadro de hemiparesia en relación con embolismos múltiples en la arteria cerebral media contralateral al aneurisma, aunque no pudo demostrarse definitivamente la relación entre el fármaco y el evento trombótico ${ }^{111}$.

El aFVIIr se ha empleado como tratamiento preventivo en una serie de 18 clipajes aneurismáticos ${ }^{104}$. Pese a la ausencia de rotura y sangrado intraoperatorio, 7 pacientes presentaron complicaciones trombóticas venosas menores, lo que cuestiona la indicación. No hay otras evidencias en esta población salvo la utilización ocasional en un paciente con múltiples aneurismas y déficit de $\mathrm{FVII}^{120} \mathrm{o}$ en pacientes con coagulopatía asociada ${ }^{118}$.

La escisión neuroquirúrgica de las anomalías cerebrovasculares a menudo se acompaña de un riesgo hemorrágico significativo, por lo que el aFVIIr puede ser un adyuvante efectivo en el control de la hemorragia quirúrgica ${ }^{23,37}$.

En neurorradiología intervencionista la rotura iatrogénica vascular por un microcatéter o las espirales (coils) es poco frecuente, pero cuando aparece presenta elevada morbi-mortalidad. Entre las nuevas opciones de tratamiento de esta complicación se encuentra la administración "focal" de aFVIIr a través del microcatéter que luego se retira y excluye. También, en caso de ruptura aneurismática durante la embolización, el rFVII se ha utilizado de forma sistémica para la reversión rápida y precoz de los antiagregantes plaquetarios (aspirina, tienopiridinas y antagonistas del receptor IIb/IIIa plaquetario, por ejemplo abciximab) administrados previamente a la embolización ${ }^{55,73,147}$.

\section{c. Procedimientos neuroquirúrgicos, no vasculares, elec- tivos}

Un metaanálisis reciente realizado en diversas cirugías, que no incluía pacientes neuroquirúrgicos, mostró una reducción del consumo de hemoderivados en los pacientes que recibieron aFVIIr de forma profiláctica, especialmente con dosis $>50 \mu \mathrm{g} \cdot \mathrm{Kg}^{-1}$, sin un incremento relevante de los efectos secundarios del fármaco ${ }^{114}$; aunque los autores recomiendan la realización de nuevos estudios para confirmar los resultados y su posible impacto económico. A la vista de estos resultados, se ha recurrido de forma esporádica al aFVIIr en procedimientos neuroquirúrgicos, aunque su empleo en estas circunstancias no se aconseje ${ }^{86}$.

Se han publicado al menos un caso aislado ${ }^{131}$ y 3 series del uso de aFVIIr en poblaciones pediátricas. Una de ellas describe el empleo del fármaco con resultados satisfactorios en la neurocirugía tumoral en 8 niños ${ }^{59}$; otra incluye dos glioblastomas multiformes ${ }^{54}$ y la tercera relata el empleo en tres craneosinostosis, una lesión traumática y un tumor cerebral ${ }^{139}$.

En adultos, las series publicadas describen el tratamiento en 3 pacientes sometidos a exéresis tumorales y sangrado intraoperatorio tras agotar las medidas convencionales ${ }^{66}$. Otra describe el control de la hemorragia intraoperatoria grave en 2 pacientes ${ }^{25}$ con tratamiento más precoz, uno de los cuales recibía clopidogrel y ácido acetilsalicílico antes de la intervención. Se han comunicado éxitos terapéuticos aislados en hemorragias relacionadas con un hemangiopericitoma ${ }^{46}$, en una reintervención por sangrado tras exéresis de un hemangioblastoma cerebeloso ${ }^{62}$, o como profilaxis del sangrado en un paciente con déficit de factor $\mathrm{XI}^{133}$.

De forma esporádica, se ha comunicado la eficacia del fármaco en el control de la hemorragia, asociada a la coagulopatía dilucional, en la cirugía de la fusión espinal por escoliosis infantil ${ }^{137}$, donde podría ser una indicación adecuada $^{86}$. Los resultados de un ensayo clínico aleatorio sobre seguridad del aFVIIr, administrado en 3 dosis de 30,60 y $120 \mu \mathrm{g} \bullet \mathrm{Kg}^{-1}$, realizado en 49 pacientes con esta patología, no han demostrado aumento de complicaciones trombóticas, pero sí una reducción del sangrado y el requerimiento transfusional ${ }^{119}$, aunque el pequeño tamaño muestral no permite extraer conclusiones definitivas.

\section{d. Politraumatismo y neurotrauma}

En 1999 Kenet G. et als ${ }^{69}$ describieron el uso de aFVIIr para el control de hemorragia incoercible en un soldado con herida por arma de fuego. A partir de este relato se inició su aplicación terapéutica para el manejo de la hemorragia 
crítica aguda de diferente etiología. En la guerra de Irak se utilizó el aFVIIr en 300 soldados siguiendo una pauta de dos dosis de $90 \mu \mathrm{g} \cdot \mathrm{Kg}^{-1}$, una al llegar al servicio de emergencias y otra al controlar la hipotermia. En esta experiencia militar se concluyó que el aFVIIr puede reducir la hemorragia y los requerimientos de transfusión en heridas de guerra ${ }^{15,52,121}$.

Hasta un $14 \%$ de pacientes con traumatismo craneoencefálico (TCE) grave ingresan con coagulopatía ${ }^{125,127}$. En un ensayo aleatorio prospectivo realizado para valorar seguridad, con dosis crecientes de aFVIIr frente a placebo, en 97 pacientes con contusiones cerebrales traumáticas, se observó una tendencia a menor aumento de la lesión hemorrágica a las 72 horas con el fármaco (con dosis $\geq 80$ $\mu \mathrm{g} \cdot \mathrm{Kg}^{-1}$ ) y un incremento no significativo en el número de eventos trombóticos (fundamentalmente trombosis venosa asintomática), sin diferencias en la mortalidad ${ }^{102}$. La utilización en esta indicación está pendiente también de resultados más concluyentes.

El resto de evidencias sobre seguridad y eficacia del fármaco en el TCE se basa en series retrospectivas. Se ha empleado para revertir coagulopatías ${ }^{100,125}$ que parecen aumentar la mortalidad ${ }^{96}$; para evitar la progresión de las lesiones hemorrágicas ${ }^{149}$; para reducir el riesgo hemorrágico antes de la colocación de drenajes ventriculares externos y en el control del sangrado profuso de los pacientes que requieren intervenciones urgentes ${ }^{9}$. En uno de estos estudios el empleo de aFVIIr se asoció a un menor tiempo hasta la corrección de la coagulopatía, lo que permitió la intervención quirúrgica más precoz y un menor consumo de plasma, sin diferencias en la mortalidad ${ }^{125}$. El mismo grupo ha publicado un estudio retrospectivo, en el que el tratamiento con aFVIIr a pacientes con TCE asociado a coagulopatía e International Normalized Ratio (INR) $\geq 1,4$ redujo la estancia en intensivos y el coste del proceso, respecto a un grupo que sólo corrigió su coagulopatía con medidas convencionales, sin incremento de efectos adversos y sin diferencias en la mortalidad ${ }^{126}$.

Con estos antecedentes, la valoración de administrar aFVIIr en el neurotrauma debe realizarse considerando la existencia de coagulopatía, el tipo de sangrado, el riesgo de expansión de hematoma y la urgencia de una hipotética cirugía ${ }^{9,100}$.

aFVIIr en la reversión de la anticoagulación oral, antiagregación plaquetaria, trombolíticos y coagulopatías de los pacientes neurocríticos

Cada día más pacientes son tratados con anticoagulantes dicumarínicos (acenocumarol, warfarina) por diversas patologías, fundamentalmente la fibrilación auricular y prótesis cardiacas $^{33}$. La HIC en relación con la anticoagulación supone un $10-12 \%$ del total ${ }^{31}$. Su riesgo de desarrollo se sitúa en torno al 1-2\% y suele presentarse con un volumen mayor, más duración y peor pronóstico que la no relacionada con la anticoagulación ${ }^{7}$.

La reversión espontánea de los antagonistas de la vitamina $\mathrm{K}$ requiere de unos 4 días $^{79}$; sin embargo los pacientes tratados con esta medicación que ingresan con una hemorragia intracraneal o un hematoma raquimedular ${ }^{136}$ son una emergencia médica, requieran o no intervención quirúrgica urgente ${ }^{43}$, y precisan una reversión rápida, precoz y eficaz de la anticoagulación ${ }^{2}$; ya que de lo contrario la mortalidad y/o las secuelas neurológicas son elevadas ${ }^{20}$. La coagulación debe restaurarse a unos niveles de INR inferiores a $1,2-1,4^{9,10} \mathrm{o}$ incluso $1,0^{112}$, aunque el objetivo verdadero sea restituir la adecuada generación de trombina ${ }^{79}$.

La reversión se realiza en función de la gravedad y urgencia de la hemorragia y del INR al ingreso, mediante vitamina $\mathrm{K}$, plasma fresco congelado, $\mathrm{CCP}$ o aFVIIr, aunque generalmente se emplean combinaciones de $\operatorname{varios}^{31,81,122}$. Debe recordarse que estas patologías presentan elevado potencial trombótico por la inmovilidad prolongada de los pacientes ${ }^{56}$ y porque suelen ser ancianos, con alta incidencia de hipertensión, diabetes y ateroesclero$\operatorname{sis}^{34}$.

Erhardtsen et al en 1998 fueron los primeros en describir que el aFVIIr revertía rápidamente el efecto de los anticoagulantes orales de una manera dosis-dependiente ${ }^{38}$. Los defensores del aFVIIr para esta indicación remarcan que se consigue la reversión del INR durante 2 horas con dosis de $5 \mu \mathrm{g} \cdot \mathrm{Kg}^{-1}$ (y de hasta $24 \mathrm{~h}$ con dosis de $120 \mu \mathrm{g} \bullet \mathrm{Kg}^{-}$ $\left.{ }^{1}\right)^{41}$, que la reversión se realiza de forma precoz ${ }^{63,103,116,118} \mathrm{y}$ que evita la transfusión de hemoderivados con sus riesgos y costes asociados ${ }^{108}$. No obstante, con frecuencia, el aFVIIr debe suplementarse con vitamina $\mathrm{K}$ o plasma ${ }^{103}$ porque no repone otros factores y su vida media es corta ${ }^{2}$.

Una serie retrospectiva muestra una tendencia a la mejor recuperación funcional en una población neurocrítica mixta (hemorragias espontáneas, hematomas traumáticos, clipajes y embolizaciones aneurismáticas) tratada con aFVIIr para reversión de la coagulopatía, que un grupo control histórico tratado con medidas convencionales, además de una reversión más precoz y eficaz de la coagulopatía ${ }^{118}$.

Una reciente revisión sugiere que para la reversión del acenocumarol se dispone de agentes eficaces sin requerir el aFVIIr, excepto en el caso concreto de la HIC del adulto si se administra dentro de las 4 horas de iniciados los sínto$\mathrm{mas}^{86}$. No obstante, cualquiera de los métodos que se elija, presenta ventajas e inconvenientes, que se resumen en la Tabla 2, por lo que no existe una guía clínica con evidencias sólidas y las recomendaciones actuales se basan en series pequeñas de casos, retrospectivas y con muchos sesgos potenciales ${ }^{4}$. Incluso la opinión de un grupo de expertos en el tema arrojó opiniones muy variadas ${ }^{2}$. Las anteriormente citadas guías de la AHA concluyen que el aFVIIr no repone 
Tabla 2

Ventajas e inconvenientes de los métodos de reversión intravenosos de la anticoagulación en pacientes con patología neurocrítica (adaptado de $\mathrm{de}^{2,20,31,43,77,79,96,97,112,122}$ )

\begin{tabular}{|l|c|c|l|l|}
\hline & Dosis & Inicio de acción & Ventajas & \multicolumn{1}{|c|}{ Inconvenientes } \\
\hline Vit. K & $5-10 \mathrm{mg}$ & $6-24 \mathrm{~h}$ & Escasos efectos adversos. & $\begin{array}{l}\text { Inadecuado como agente único } \\
\text { si se requiere reversión rápida. } \\
\text { Riesgo raro de anafilaxia (3/10000). } \\
\text { Ineficaz en insuficiencia hepática. } \\
\text { Bloqueo de la anticoagulación hasta una } \\
\text { semana si se requiere reinicio }\end{array}$ \\
\hline PFC & $20-25 \mathrm{mg}$ & $4-6 \mathrm{~h}$ & Precio. & $\begin{array}{l}\text { Requiere tiempo (tests + administración). } \\
\text { Eficacia variable. }\end{array}$ \\
\begin{tabular}{|l} 
Sobrecarga de volumen (riesgo de \\
edema cerebral y/o pulmonar).
\end{tabular} \\
\begin{tabular}{|l} 
Reacciones transfusionales. \\
Riesgo transmisión vírica.
\end{tabular}
\end{tabular}

Vit.K: Vitamina K. PFC: plasma fresco congelado. CCP: concentrado de complejo protrombínico. aFVIIr: factor VII activado recombinante. FIX: factor IX.

todos los factores de coagulación y aunque el INR suele reducirse, la hemostasia puede no ser restaurada; de ahí que no se recomienda como fármaco único para la reversión de la anticoagulación en pacientes con HIC (recomendación clase III, nivel de evidencia C) ${ }^{101}$.

En el caso de HIC y tratamiento previo con antiagregantes plaquetarios se propone la transfusión de plaquetas, aunque tanto la desmopresina $\left(0,15-0,3 \mathrm{mg} \cdot \mathrm{kg}^{-1}\right)$, que aumenta los niveles de factor endotelial de von Willebrand $^{112}$, como el aFVIIr pueden tener un papel en contrarrestar la coagulopatía por estos agentes ${ }^{96}$.

Los anticoagulantes inhibidores directos de la trombina, tanto bivalentes (hirudina, lepirudina, bivaluridina) como univalentes (argatroban, dabigatran), se emplean en casos de anticoagulación y presencia de anticuerpos antiplaquetarios. No se dispone de antídotos; por lo que se sugiere, en caso de necesidad de reversión, la desmopresina, el concentrado de factor de von Willebrand, e incluso el aFVIIr ${ }^{12,145}$.

Con respecto a los fármacos trombolíticos (fibrinolíticos), se ha descrito el empleo del aFVIIr, asociado a plaquetas y crioprecipitado tras la conversión hemorrágica de un ictus isquémico, en un paciente tratado con activador tisular del plasminógeno. La evolución fue negativa, ya que el paciente desarrolló múltiples infartos isquémicos tras la administración del aFVIIr, descartándose el origen cardiaco de los émbolos y falleció en la $2^{\mathrm{a}}$ semana de evolución ${ }^{80}$.

En otro tipo de coagulopatías, por ejemplo cirrosis, déficit adquirido de FVII; también hay casos descritos de tratamiento con aFVIIr, con el resultado de una mejora rápida de la hemostasia y estabilización del hematoma cerebral $^{62,98}$. Una publicación reciente describe el empleo de $3 \mathrm{mg}$ de aFVIIr, sin complicaciones hemorrágicas ni trombóticas, en 10 pacientes con encefalopatía grado IIIIV secundaria a fallo hepático fulminante e INR $>3$, antes de la colocación de un sensor de presión intracraneal intraparenquimatoso ${ }^{74}$.

Por último, pacientes con neoplasia extracraneal conocida, trombocitopenia, o trastornos de la hemostasia en cirugías previas, pueden ser susceptibles al desarrollo de coagulopatía, aún con un INR normal. En 2 de estos casos que requerían cirugía urgente se utilizó el aFVIIr con buen resultado de eficacia y funcional ${ }^{94}$. 


\section{Cuestiones controvertidas en la terapia con aFVIIr}

\section{a. ¿Cuál es el momento ideal de tratamiento?}

La precocidad parece ser fundamental para la efica$\mathrm{cia}^{77,88}$, especialmente en lesiones cerebrales ${ }^{10,97}$. Se podría recomendar su empleo una vez que fallan las medidas convencionales, pero sin que la demora haga que su eficacia se reduzca ${ }^{10,77,94}$. Para aumentar dicha eficacia, además de optimizarse el recuento plaquetario, el $\mathrm{pH}$ y la temperatura, debe intentarse mantener un hematocrito por encima de $24 \%$ y un nivel superior a $50 \mathrm{mg} \cdot \mathrm{dL}^{-1} \mathrm{de}$ fibrinógeno ${ }^{86,88,148}$.

\section{b. ¿Cuál es la dosis óptima en caso de administración a estos pacientes?}

Las recomendaciones en las indicaciones off-labe ${ }^{86}$ son de $40-90 \mu \mathrm{g} \cdot \mathrm{Kg}^{-1}$. La menor dosis eficaz comunicada del fármaco ha sido de $5 \mu \mathrm{g} \cdot \mathrm{Kg}^{-1}$. En el empleo como profilaxis para situaciones de déficit de factores, la dosis recomendada $^{75,82,133,146}$ se sitúa en el rango de los $15-60 \mu \mathrm{g} \cdot \mathrm{Kg}^{-1}$, y para la hemofilia es de $90 \mu \mathrm{g} \cdot \mathrm{Kg}^{-1}$, que ha sido la empleada inicialmente en el tratamiento de la coagulopatía asociada con la hemorragia masiva y la politransfusión. Recomendaciones más recientes sugieren dosis mayores para esta indicación (100-140 $\left.\mu \mathrm{g} \cdot \mathrm{Kg}^{-1}\right)$, administradas en 2 a 5 minutos $^{88}$. Puede repetirse la administración en función de la eficacia clínica, ya que su vida media es corta (2,3 horas). La necesidad de repetición es otro inconveniente, aunque se requiere sobre todo en pacientes hemofílicos ${ }^{41}$.

\section{c. ¿Cuál es el método ideal de monitorización antes y des- pués de la administración del fármaco?}

Los tests rutinarios de coagulación, además de que no evalúan adecuadamente la fibrinolisis y la función plaquetaria ${ }^{10}$, no son adecuados para valorar el estado de la hemostasia ${ }^{5,70}$. La normalización del tiempo de protrombina (TP) es habitual tras la administración del fármaco, lo que no siempre se acompaña de un adecuado control del sangrado $^{5}$. Ni siquiera la medición del nivel de FVII en sangre es adecuado ${ }^{123}$. El tromboelastograma puede ser de ayuda ${ }^{60}$, aunque el mejor indicador de eficacia sigue siendo el cese del sangrado ${ }^{41}$ y el descenso en las necesidades transfusionales ${ }^{33,88,139}$.

\section{d. Administración de aFVIIr en poblaciones especiales: obstetricia y pediatría}

El aFVIIr se ha utilizado en poblaciones pediátricas, aunque con evidencias escasas y contradictorias ${ }^{19,86,100}$. Los niños deberían presentar bajo riesgo tromboembólico porque no tienen ateroesclerosis generalizada ${ }^{5}$, aunque su sistema fibrinolítico es inmaduro y podría asociarse a coagulación intravascular diseminada (CID $)^{59}$. En cuanto a la embarazada/puérpera, pese al elevado potencial trombogé- nico de esta población, la hemorragia obstétrica ${ }^{3}$ supone en torno al 5\% de las indicaciones "off-label", con una eficacia en la reducción del sangrado del $76 \%$ y una tasa de complicaciones trombóticas del 2-3\% ${ }^{110}$. No obstante, también se necesitan ensayos clínicos para estas poblaciones.

\section{e. ¿Justifica el coste del fármaco el beneficio que se obtiene?}

El elevado precio del fármaco obliga a evaluar especialmente las indicaciones "off-label", que deberían individualizarse, al menos mientras no haya mejores evidencias al respecto $^{14,86,113}$.

Un análisis fármaco-económico en pacientes críticos, muestra que la administración del aFVIIr podría ser eficiente cuando se requieren 14-19 concentrados de hematíes, aunque el estudio es retrospectivo y se basa en un modelo matemático ${ }^{83}$. En otra serie retrospectiva en la que se trataron 18 pacientes con $90 \mu \mathrm{g} \cdot \mathrm{Kg}^{-1}$ antes de una intervención urgente para drenar hematomas subdurales con coagulopatía (INR>1,2), el aFVIIr supuso el $8 \%$ del gasto hospitalario, pero los autores justifican el empleo en la reducción del tiempo de espera para la cirugía, la elevada eficacia y el hecho de evitar tratamientos alternativos ${ }^{97}$.

Cuando se emplea a dosis bajas para la reversión de la anticoagulación $\left(15-20 \mu \mathrm{g} \bullet \mathrm{Kg}^{-1}\right)$, y suponiendo que no existan complicaciones trombóticas, el balance podría resultar favorable con respecto a la transfusión de plasma fresco ${ }^{33}$.

Los pacientes traumáticos o quirúrgicos pueden presentar mejores tasas de supervivencia debido a que ésta depende en muchos de esos casos del control del sangrado; mientras que en pacientes críticos médicos, la presencia de fallos orgánicos y de CID suele condicionar el pronóstico e incluso ser la responsable de la coagulopatía y el sangrado $^{5}$.

\section{Consideraciones finales}

El eptacog alfa activado es un agente procoagulante muy eficaz en determinadas situaciones clínicas, aunque no debe considerarse un agente hemostático universal. Su administración debe ajustarse a las directrices aprobadas, aunque si lo utilizamos en una indicación "off-label", requiere una valoración muy estricta de los beneficios y riesgos esperados debido a las dudas existentes sobre su perfil de seguridad y al elevado coste.

Cuando tratamos pacientes neurocríticos, beneficios esperados podrían ser: a) la elevada eficacia comunicada en el control de la hemorragia, b) la reversión rápida de una coagulopatía muy frecuente en esta población, cuando el tiempo para actuar es muy importante, c) el cese del sangrado incoercible en áreas que toleran mal pequeños incrementos de volumen y d) la reducción de efectos adversos 
asociados a otros tratamientos hemostáticos. En el debe del fármaco se sitúa el riesgo de complicaciones trombóticas, el hecho de que no siempre es eficaz sin asociar otros agentes hemostáticos y que su vida media corta puede obligar a utilizar dosis repetidas del agente; así como la elevada producción de trombina, que más allá del potencial trombótico, podría incrementar el edema cerebral.

\section{Bibliografía}

1. Aguilar, M.I., Demaerschalk, B.M.: Intracerebral hemorrhage. Semin Neurol. 2007; 27: 376-384.

2. Aguilar, M.I., Hart, R.G., Kase, C.S., Freeman, W.D., Hoeben, B.J., Garcia, R.C., et al.: Treatment of warfarin-associated intracerebral hemorrhage: literature review and expert opinion. Mayo Clin Proc. 2007; 82: 82-92.

3. Ahonen, J., Jokela, R.: Recombinant factor VIIa for lifethreatening post-partum haemorrhage. Br J Anaesth. 2005; 94 : 592-595.

4. Aiyagari, V., Testai, F.D.: Correction of coagulopathy in warfarin associated cerebral hemorrhage. Curr Opin Crit Care. 2009; 15: 87-92.

5. Alten, J.A., Benner, K., Green, K., Toole, B., Tofil, N.M., Winkler, M.K.: Pediatric off-label use of recombinant factor VIIa. Pediatrics. 2009; 123: 1066-1072.

6. Amarenco, P., Bogousslavsky, J., Caplan, L.R., Donnan, G.A., Hennerici, M.G.: Classification of stroke subtypes. Cerebrovasc Dis. 2009; 27: 493-501.

7. Anderson, C.S.: Medical management of acute intracerebral hemorrhage. Curr Opin Crit Care. 2009; 15: 93 - 98.

8. Barnes, C., Blanchette, V., Canning, P., Carcao, M.: Recombinant FVIIa in the management of intracerebral haemorrhage in severe thrombocytopenia unresponsive to platelet- enhancing treatment. Transfus Med. 2005; 15: 145150.

9. Bartal, C., Freedman, J., Bowman, K., Cusimano, M.: Coagulopathic patients with traumatic intracranial bleeding: defining the role of recombinant factor VIIa. J Trauma. 2007; 63: 725-732.

10. Bartal, C., Yitzhak, A.: The role of thromboelastometry and recombinant factor VIIa in trauma. Curr Opin Anaesthesiol. 2009; 22: 281-288 .

11. Beltran de Heredia, S., Bisbe, E., Rojo, A., Gracia, M.P., Lopez, M., Escolano, F.: Usefulness of activated recombinant factor VII for controlling massive bleeding: 4 years' experience in a university hospital. Rev Esp Anestesiol Reanim. 2008; 55: 355-359.

12. Bijsterveld, N.R., Moons, A.H., Boekholdt, S.M., van Aken, B.E., Fennema, H., Peters, R.J., et al.: Ability of recombinant factor VIIa to reverse the anticoagulant effect of the pentasaccharide fondaparinux in healthy volunteers. Circulation. 2002; 106: 2550-2554.

13. Bijsterveld, N.R., Vink, R., van Aken, B.E., Fennema,
H., Peters, R.J., Meijers, J.C., et al.: Recombinant factor VIIa reverses the anticoagulant effect of the long-acting pentasaccharide idraparinux in healthy volunteers. Br J Haematol. 2004; 124: 653-658.

14. Biss, T.T., Hanley, J.P.: Recombinant activated factor VII ( $\mathrm{rFVIIa/NovoSeven)} \mathrm{in} \mathrm{intractable} \mathrm{haemorrhage:} \mathrm{use} \mathrm{of} \mathrm{a}$ clinical scoring system to predict outcome. Vox Sang. 2006; 90: 45-52.

15. Boffard, K.D., Riou, B., Warren, B., Choong, P.I., Rizoli, S., Rossaint, R., et al.: Recombinant factor VIIa as adjunctive therapy for bleeding control in severely injured trauma patients: two parallel randomized, placebo-controlled, double-blind clinical trials. J Trauma. 2005; 59: 8-15.

16. Bogousslavsky, J., Aarli, J., Kimura, J.: Stroke: time for a global campaign? Cerebrovasc Dis. 2003; 16: 111-113.

17. Bordes, J., Asencio, Y., Kenane, N., Fesselet, J., Meaudre, E., Goutorbe, P.: Recombinant activated factor VII for acute subdural haematoma in an elderly patient taking fondaparinux. Br J Anaesth. 2008; 101: 575-576.

18. Bowles, K.M., Callaghan, C.J., Taylor, A.L., Harris, R.J., Pettigrew, G.J., Baglin, T.P., et al.: Predicting response to recombinant factor VIIa in non-haemophiliac patients with severe haemorrhage. Br J Anaesth. 2006; 97: 476-481.

19. Brady, K.M., Easley, R.B., Tobias, J.D.: Recombinant activated factor VII (rFVIIa) treatment in infants with hemorrhage. Paediatr Anaesth. 2006; 16: 1042-1046.

20. Brody, D.L., Aiyagari, V., Shackleford, A.M., Diringer, M.N.: Use of recombinant factor VIIa in patients with warfarin- associated intracranial hemorrhage. Neurocrit Care. 2005; 2: 263-267.

21. Brohi, K., Cohen, M.J., Ganter, M.T., Schultz, M.J., Levi, M., Mackersie, R.C., et al.: Acute coagulopathy of trauma: hypoperfusion induces systemic anticoagulation and hyperfibrinolysis. J Trauma. 2008; 64: 1211-1217.

22. Brown, C.V., Foulkrod, K.H., Lopez, D., Stokes, J., Villareal, J., Foarde, K., et al.: Recombinant factor VIIa for the correction of coagulopathy before emergent craniotomy in blunt trauma patients. J Trauma. 2010; 68: 348-352.

23. Buckmiller, L.M., Richter, G.T., Waner, M., Suen, J.Y.: Use of recombinant factor VIIa during excision of vascular anomalies. Laryngoscope. 2007; 117: 604-609.

24. Busani, S., Marietta, M., Pasetto, A., Girardis, M.: Use of recombinant factor VIIa in a thrombocytopenic patient with spontaneous intracerebral haemorrhage. Thromb Haemost. 2005; 93: 381-382.

25. Cassinello, O.C., Cotera, U.I., Cortes, F.S., Felix, L.J.: Intraoperative use of activated recombinant factor VII in a patient with brain cancer. Rev Esp Anestesiol Reanim. 2009; 56: 394-396.

26. Conti, S., La, T.D., Gambelunghe, G., Ciorba, E., Iorio, A.: Successful treatment with rFVIIa of spontaneous intracerebral hemorrhage in a patient with mechanical prosthetic heart valves. Clin Lab Haematol. 2005; 27: 283-285. 
27. Crozier, S.: Treatment of cerebral hemorrhage. Presse Med. 2007; 36: 142-149.

28. Cruz-Flores, S.: Myocardial injury in patients with intracerebral hemorrhage treated with recombinant factor VIIa. Neurology. 2007; 68: 1750-1751.

29. Cruz-Flores, S.: Unexpected posthemorrhagic hydrocephalus in patients treated with rFVIIa. Neurology. 2007; 68: 1084-1085.

30. Culley, C.M., Guttendorf, S., Coldren, M., Skledar, S.J., Gross, P.R., Spiro, R.M., et al.: Coagulation factor VIIa (recombinant) in nonhemophilic patients requiring neurosurgery. Am J Health Syst Pharm. 2009; 66: 1554-1559.

31. Da'as, N., Misgav, M., Kalish, Y., Varon, D.: Recombinant factor VIIa for rapid reversal of anticoagulant effect in patients with intracranial hemorrhage: the Israeli experience and review of the literature. Isr Med Assoc J. 2006; 8: 807811.

32. Davie, E.W., Ratnoff, O.D.: Waterfall sequence for intrinsic blood clotting. Science. 1964; 145: 1310-1312.

33. Deveras, R.A., Kessler, C.M.: Reversal of warfarininduced excessive anticoagulation with recombinant human factor VIIa concentrate. Ann Intern Med. 2002; 137: 884-888.

34. Diringer, M.N., Skolnick, B.E., Mayer, S.A., Steiner, T., Davis, S.M., Brun, N.C., et al.: Risk of thromboembolic events in controlled trials of rFVIIa in spontaneous intracerebral hemorrhage. Stroke. 2008; 39: 850-856.

35. Diringer, M.N., Skolnick, B.E., Mayer, S.A., Steiner, T., Davis, S.M., Brun, N.C., et al.: Thromboembolic events with recombinant activated factor VII in spontaneous intracerebral hemorrhage: results from the Factor Seven for Acute Hemorrhagic Stroke (FAST) trial. Stroke. 2010; 41: 4853.

36. Donnan, G.A., Fisher, M., Macleod, M., Davis, S.M.: Stroke. Lancet. 2008; 371: 1612-1623.

37. Engelhardt, K., Brenneis, C., Pfausler, B., Beer, R., Brossner, G., Helbok, R., et al.: rFVIIa--for acute rebleeding of a cerebral cavernous malformation. Eur J Neurol. 2007; 14 : 117-120.

38. Erhardtsen, E., Nony, P., Dechavanne, M., Ffrench, P., Boissel, J.P., Hedner, U.: The effect of recombinant factor VIIa (NovoSeven) in healthy volunteers receiving acenocoumarol to an International Normalized Ratio above 2.0. Blood Coagul Fibrinolysis. 1998; 9: 741-748.

39. Farah, R.A., Hamod, D., Melick, N., Giansily-Blaizot, M., Sallah, S.: Successful prophylaxis against intracranial hemorrhage using weekly administration of activated recombinant factor VII in a newborn with severe factor VII deficiency. J Thromb Haemost. 2007; 5: 433-434.

40. Felfernig, M., Huepfl, M.: Experience of recombinant activated factor VII (NovoSeven) in the operating theatre and intensive care unit for the management of intracranial bleeding in nonhaemophilic patients. Clin Neurol Neurosurg. 2008; 110: 227-232.
41. Fewel, M.E., Park, P.: The emerging role of recombinant- activated factor VII in neurocritical care. Neurocrit Care. 2004; 1: 19-29.

42. Flaherty, M.L., Woo, D., Haverbusch, M., Sekar, P., Khoury, J., Sauerbeck, L., et al.: Racial variations in location and risk of intracerebral hemorrhage. Stroke. 2005; 36: 934937.

43. Freeman, W.D., Brott, T.G., Barrett, K.M., Castillo, P.R., Deen, H.G., Jr., Czervionke, L.F., et al.; Recombinant factor VIIa for rapid reversal of warfarin anticoagulation in acute intracranial hemorrhage. Mayo Clin Proc. 2004; 79: 1495-1500.

44. Gando, S.: Disseminated intravascular coagulation in trauma patients. Semin Thromb Hemost. 2001; 27: 585-592.

45. Gerald, A.G.: Update on hemostasis: neurosurgery. Surgery. 2007; 142(4 Suppl): S55-S60.

46. Gerlach, R., Marquardt, G., Wissing, H., Scharrer, I., Raabe, A., Seifert, V.: Application of recombinant activated factor VII during surgery for a giant skull base hemangiopericytoma to achieve safe hemostasis. Case report. J Neurosurg. 2002; 96: 946-948.

47. Greenberg, S.M.: Is "compassionate use" compassionate?: rFVIIa for intracerebral hemorrhage. Neurology. 2006; 67: 934-935.

48. Grysiewicz, R.A., Thomas, K., Pandey, D.K.: Epidemiology of ischemic and hemorrhagic stroke: incidence, prevalence, mortality, and risk factors. Neurol Clin. 2008; 26: 871-895.

49. Guyton, C.A., Hall, J.E.: Hemostasis and Blood Coagulation. En: Guyton \& Hall: Textbook of Medical Physiology. 11th edición. New York: Elsevier Saunders, W.B.; 2005. p 457-68.

50. Hallevi, H., Gonzales, N.R., Barreto, A.D., MartinSchild, S., Albright, K.C., Noser, E.A., et al.: The effect of activated factor VII for intracerebral hemorrhage beyond 3 hours versus within 3 hours. Stroke. 2008; 39: 473-475.

51. Hansen, T.W.; Prophylaxis of intraventricular hemorrhage in premature infants: new potential tools, new potential challenges. Pediatr Crit Care Med. 2006; 7: 90-92.

52. Harrison, T.D., Laskosky, J., Jazaeri, O., Pasquale, M.D., Cipolle, M.: "Low-dose" recombinant activated factor VII results in less blood and blood product use in traumatic hemorrhage. J Trauma. 2005; 59: 150-154.

53. Hartmann, M., Sucker, C.: Pharmacology and clinical use of recombinant activated factor seven in neurosciences. Neurocrit Care. 2007; 6: 149-157.

54. Hartmann, M., Sucker, C., Messing, M.: Recombinant activated factor VII in the treatment of near-fatal bleeding during pediatric brain tumor surgery. Report of two cases and review of the literature. J Neurosurg. 2006; 104 (1 Suppl): 5558.

55. Hashimoto, T., Gupta, D.K., Young, WL.: Interventional neuroradiology--anesthetic considerations. Anesthesiol 
Clin North America. 2002; 20: 347-359.

56. Hawryluk, G.W., Cusimano, M.D.: The role of recombinant activated factor VII in neurosurgery: hope or hype? J Neurosurg. 2006; 105: 859-868.

57. Hedner, U.: Mechanism of action of factor VIIa in the treatment of coagulopathies. Semin Thromb Hemost. 2006; 32 (Suppl 1): 77-85.

58. Hedner, U., Brun, N.C.: Recombinant factor VIIa (rFVIIa): its potential role as a hemostatic agent. Neuroradiology. 2007; 49: 789-793.

59. Heisel, M., Nagib, M., Madsen, L., Alshiekh, M., Bendel, A.: Use of recombinant factor VIIa (rFVIIa) to control intraoperative bleeding in pediatric brain tumor patients. Pediatr Blood Cancer. 2004; 43: 703-705.

60. Hirasaki, Y., Suematsu, Y., Yasuda, T., Tajima, K..: Thromboelastometry to guide recombinant activated factor VII therapy for postoperative refractory intracranial bleeding. Anesth Analg. 2010; 110: 261-262.

61. Hoffman, M.: Remodeling the blood coagulation cascade. J Thromb Thrombolysis. 2003;16 :17-20.

62. Huang, W.Y., Kruskall, M.S., Bauer, K.A., Uhl, L., Shaz, B.H.: The use of recombinant activated factor VII in three patients with central nervous system hemorrhages associated with factor VII deficiency. Transfusion. 2004; 44:15621566.

63. Ilyas, C., Beyer, G.M., Dutton, R.P., Scalea, T.M., Hess, J.R.: Recombinant factor VIIa for warfarin-associated intracranial bleeding. J Clin Anesth. 2008; 20: 276-279.

64. Ionita, C.C., Ferrara, J., McDonagh, D.L., Grossi, P., Graffagnino, C.: Systemic hemostasis with recombinantactivated factor VII followed by local thrombolysis with recombinant tissue plasminogen activator in intraventricular hemorrhage. Neurocrit Care. 2005; 3: 246-248.

65. Kapapa, T., Konig, K., Heissler, H.E., Schatzmann, C., Tschan, C.A., Perl, M., et al.: The use of recombinant activated factor VII in neurosurgery. Surg Neurol. 2009; 71: 172-179.

66. Karadimov, D., Binev, K., Nachkov, Y., Platikanov, V.: Use of activated recombinant Factor VII (NovoSeven) during eurosurgery. J Neurosurg Anesthesiol. 2003; 15: 330-332.

67. Karimi, M.: Successful control of central nervous system bleeding in two newborns with severe factor VII deficiency using rFVIIa administered via Port-a-Cath. Semin Hematol. 2008; 45 (2 Suppl 1): S74.

68. Kelley, R.E., Borazanci, A.P.: Stroke rehabilitation. Neurol Res. 2009; 31: 832-840.

69. Kenet, G., Walden, R., Eldad, A., Martinowitz, U.: Treatment of traumatic bleeding with recombinant factor VIIa. Lancet. 1999; 354: 1879.

70. Key, N.S.: Recombinant FVIIa for intractable hemorrhage: more questions than answers. Transfusion. 2003; 43: 1649-1651.

71. Kissela, B.M., Eckman, M.H.: Cost effectiveness of recombinant factor VIIa for treatment of intracerebral hemorr- hage. BMC Neurol. 2008; 8: 1-9.

72. Kluger, Y., Riou, B., Rossaint, R., Rizoli, S.B., Boffard, K.D., Choong, P.I., et al.: Safety of rFVIIa in hemodynamically unstable polytrauma patients with traumatic brain injury: post hoc analysis of 30 patients from a prospective, randomized, placebo-controlled, double-blind clinical trial. Crit Care. 2007; 11: R85.

73. Lakhani, S., Guha, A., Nahser, H.C.: Anaesthesia for endovascular management of cerebral aneurysms. Eur J Anaesthesiol. 2006; 23: 902-913.

74. Le, T.V., Rumbak, M.J., Liu, S.S., Alsina, A.E., van L,H., Agazzi, S.: Insertion of intracranial pressure monitors in fulminant hepatic failure patients: early experience using recombinant factor VII. Neurosurgery. 2010; 66: 455-458.

75. Leal, R., Alberca, I., Asuero, M.S., Boveda, J.L., Carpio, N., Contreras, E., et al.: The $<<$ Seville $>>$ Consensus Document on Alternatives to Allogenic Blood Transfusion. Med Clin (Barc ). 2006; 127 (Supl.1): 3-20.

76. Levi, M.: Recombinant factor VIIa: a general hemostatic agent? Not yet. J Thromb Haemost. 2004; 2: 1695-1697.

77. Levi, M., Peters, M., Buller, H.R.: Efficacy and safety of recombinant factor VIIa for treatment of severe bleeding: a systematic review. Crit Care Med. 2005; 33: 883-890.

78. Levy, J.H., Fingerhut, A., Brott, T., Langbakke, I.H., Erhardtsen, E., Porte, R.J.: Recombinant factor VIIa in patients with coagulopathy secondary to anticoagulant therapy, cirrhosis, or severe traumatic injury: review of safety profile. Transfusion. 2006; 46: 919-933.

79. Levy, J.H., Tanaka, K.A., Dietrich, W.: Perioperative hemostatic management of patients treated with vitamin $\mathrm{K}$ antagonists. Anesthesiology. 2008; 109: 918-926.

80. Libman, R.B., Lungu, C., Kwiatkowski, T.: Multiple ischemic strokes associated with use of recombinant activated factor VII. Arch Neurol. 2007; 64: 879-881.

81. Lin, J., Hanigan, W.C., Tarantino, M., Wang, J.: The use of recombinant activated factor VII to reverse warfarininduced anticoagulation in patients with hemorrhages in the central nervous system: preliminary findings. J Neurosurg. 2003; 98: 737-740.

82. Liu, N., Aldea, S., Francois, D., Cherqui-Michel, M., Giansily-Blaizot, M., Fischler, M.: Recombinant activated factor VII for a patient with factor VII deficiency undergoing urgent intracerebral haematoma evacuation with underlying cavernous angioma. Br J Anaesth. 2009; 103: 858-860.

83. Loudon, B., Smith, M.P.: Recombinant factor VIIa as an adjunctive therapy for patients requiring large volume transfusion: a pharmacoeconomic evaluation. Intern Med J. 2005; 35: 463-467.

84. MacFarlane, R.G.: An enzyme cascade in the blood clotting mechanism, and its function as a biochemical amplifier. Nature. 1964; 202: 498-499.

85. Mackman, N.: The role of tissue factor and factor VIIa in hemostasis. Anesth Analg. 2009; 108: 1447-1452. 
86. Mallarkey, G., Brighton, T., Thomson, A., Kaye, K., Seale, P., Gazarian, M.: An evaluation of eptacog alfa in nonhaemophiliac conditions. Drugs. 2008; 68: 1665-1689.

87. Marshall, J.C.: Inflammation, coagulopathy, and the pathogenesis of multiple organ dysfunction syndrome. Crit Care Med. 2001; 29 (7 Suppl): S99-106.

88. Martinowitz, U., Michaelson, M.: Guidelines for the use of recombinant activated factor VII (rFVIIa) in uncontrolled bleeding: a report by the Israeli Multidisciplinary rFVIIa Task Force. J Thromb Haemost. 2005; 3: 640-648.

89. Mayer, S.A.: Ultra-early hemostatic therapy for intracerebral hemorrhage. Stroke. 2003; 34: 224-229.

90. Mayer, S.A.: Complications in patients with intracerebral hemorrhage treated with recombinant factor VIIa. Neurology. 2007; 69: 319-320.

91. Mayer, S.A., Brun, N.C., Begtrup, K., Broderick, J., Davis, S., Diringer, M.N., et al.: Recombinant activated factor VII for acute intracerebral hemorrhage. N Engl J Med. 2005; 352: 777-785.

92. Mayer, S.A., Brun, N.C., Begtrup, K., Broderick, J., Davis, S., Diringer, M.N., et al.: Efficacy and safety of recombinant activated factor VII for acute intracerebral hemorrhage. N Engl J Med. 2008; 358: 2127-2137.

93. Mayer, S.A., Brun, N.C., Broderick, J., Davis, S.M., Diringer, M.N., Skolnick, B.E., et al.: Recombinant activated factor VII for acute intracerebral hemorrhage: US phase IIA trial. Neurocrit Care. 2006; 4: 206-214.

94. McClelland, S., III, Won, E.K., Lam, C.H.: Utilization of recombinant activated factor VII for intracranial hematoma evacuation in coagulopathic nonhemophilic neurosurgical patients with normal international normalized ratios. Neurocrit Care. 2007; 7 : 136-139.

95. McCrea, H.J., Ment, L.R.: The diagnosis, management, and postnatal prevention of intraventricular hemorrhage in the preterm neonate. Clin Perinatol. 2008; 35: 777-792.

96. McMillian, W.D., Rogers, F.B.: Management of prehospital antiplatelet and anticoagulant therapy in traumatic head injury: a review. J Trauma. 2009; 66: 942-950.

97. McQuay, N., Jr., Cipolla, J., Franges, E.Z., Thompson, G.E.: The use of recombinant activated factor VIIa in coagulopathic traumatic brain injuries requiring emergent craniotomy: is it beneficial? J Neurosurg. 2009; 111: 666-671.

98. Mindikoglu, A.L., Anantharaju, A., George, M., Shah, N.N., Villanueva, J., Van Thiel, D.H.: Acute intracranial hemorrhage in a cirrhotic controlled with recombinant factor VIIa. Dig Dis Sci. 2003; 48: 1130-1135.

99. Morawitz, P.: Die chemie der Blutgerinnung. Ergebn Physiol. 1905; 4: 307-423.

100. Morenski, J.D., Tobias, J.D., Jimenez, D.F.: Recombinant activated factor VII for cerebral injury-induced coagulopathy in pediatric patients. Report of three cases and review of the literature. J Neurosurg. 2003; 98: 611-316.

101. Morgenstern, L.B., Hemphill, J.C., III, Anderson,
C., Becker, K., Broderick, J.P., Connolly, E.S., Jr., et al.: Guidelines for the management of spontaneous intracerebral hemorrhage: a guideline for healthcare professionals from the American Heart Association/American Stroke Association. Stroke. 2010; 41: 2108-2129.

102. Narayan, R.K., Maas, A.I., Marshall, L.F., Servadei, F., Skolnick, B.E., Tillinger, M.N.: Recombinant factor VIIA in traumatic intracerebral hemorrhage: results of a dose-escalation clinical trial. Neurosurgery. 2008; 62: 776-786.

103. Nishijima, D.K., Dager, W.E., Schrot, R.J., Holmes, J.F.: The efficacy of factor VIIa in emergency department patients with warfarin use and traumatic intracranial hemorrhage. Acad Emerg Med. 2010; 17: 244-251.

104. Nussbaum, E.S., Janjua, T.M., Defillo, A., Sinner, P., Zelensky, A.: Perioperative use of recombinant factor VII to prevent intraoperative aneurysm rupture in high risk patients: a preliminary safety evaluation. Neurocrit Care. 2009; 10: 5560.

105. Nyquist, P.: Management of acute intracranial and intraventricular hemorrhage. Crit Care Med. 2010; 38: 946953.

106. O’Connell, K.A., Wood, J.J., Wise, R.P., Lozier, J.N., Braun, M.M.: Thromboembolic adverse events after use of recombinant human coagulation factor VIIa. JAMA. 2006; 295: 293-298.

107. Paramo Fernandez, J.A., Orbe, L.J.: The hemostatic system in the acute coronary syndromes: physiopathological and therapeutic implications. Rev Clin Esp. 2004; 204: 221227.

108. Park, P., Fewel, M.E., Garton, H.J., Thompson, B.G., Hoff, J.T.: Recombinant activated factor VII for the rapid correction of coagulopathy in nonhemophilic neurosurgical patients. Neurosurgery. 2003; 53: 34-38.

109. Pathak, A., Dutta, S., Marwaha, N., Singh, D., Varma, N., Mathuriya, S.N.: Change in tissue thromboplastin content of brain following trauma. Neurol India. 2005; 53: 178-182.

110. Phillips, L.E., McLintock, C., Pollock, W., Gatt, S.,Popham, P., Jankelowitz, G., et al.: Recombinant activated factor VII in obstetric hemorrhage: experiences from the Australian and New Zealand Haemostasis Registry. Anesth Analg. 2009; 109: 1908-1915.

111. Pickard, J.D., Kirkpatrick, P.J., Melsen, T., Andreasen, R.B., Gelling, L., Fryer, T., et al.: Potential role of NovoSeven in the prevention of rebleeding following aneurysmal subarachnoid haemorrhage. Blood Coagul Fibrinolysis. 2000; 11 (Suppl 1): S117-S120.

112. Powner, D.J., Hartwell, E.A., Hoots, W.K.: Counteracting the effects of anticoagulants and antiplatelet agents during neurosurgical emergencies. Neurosurgery. 2005; 57: 823-831.

113. Pugh, R., Wenstone, R.: Predicting response to recombinant factor VIIa in non-haemophiliac patients with severe haemorrhage. Br J Anaesth. 2007; 98: 690-691. 
114. Ranucci, M., Isgro, G., Soro, G., Conti, D., De, T.B.: Efficacy and safety of recombinant activated factor vii in major surgical procedures: systematic review and metaanalysis of randomized clinical trials. Arch Surg. 2008; 143: 296304.

115. Roberts, H.R.: Recombinant factor VIIa: a general hemostatic agent? Yes. J Thromb Haemost. 2004; 2) :16911694.

116. Robertson, J.D.: Prevention of intraventricular haemorrhage: a role for recombinant activated factor VII? J Paediatr Child Health. 2006; 42: 325-331.

117. Robinson, M.T., Rabinstein, A.A., Meschia, J.F., Freeman, W.D.: Safety of recombinant activated factor VII in patients with warfarin-associated hemorrhages of the central nervous system. Stroke. 2010; 41: 1459-1463.

118. Roitberg, B., Emechebe-Kennedy, O., min-Hanjani, S., Mucksavage, J., Tesoro, E.: Human recombinant factor VII for emergency reversal of coagulopathy in neurosurgical patients: a retrospective comparative study. Neurosurgery. 2005; 57: 832-836.

119. Sachs, B., Delacy, D., Green, J., Graham, R.S., Ramsay, J., Kreisler, N., et al.: Recombinant activated factor VII in spinal surgery: a multicenter, randomized, doubleblind, placebo-controlled, dose-escalation trial. Spine (Phila $\mathrm{Pa}$ 1976). 2007; 32: 2285-2293.

120. Schilling, A.M., Heidenreich, J.O., Oldenburg, A.C., Pietila, T., Stendel, R., Wolf, KJ.: Multiple cerebral aneurysms in factor VII deficiency. AJNR Am J Neuroradiol. 2004; 25 : 784-786.

121. Sebesta, J.: Special lessons learned from Iraq. Surg Clin North Am. 2006; 86: 711-726.

122. Sorensen, B., Johansen, P., Nielsen, G.L., Sorensen, J.C., Ingerslev, J.: Reversal of the International Normalized Ratio with recombinant activated factor VII in central nervous system bleeding during warfarin thromboprophylaxis: clinical and biochemical aspects. Blood Coagul Fibrinolysis. 2003; 14: 469-477.

123. Spahn, D.R., Tucci, M.A., Makris, M.: Is recombinant FVIIa the magic bullet in the treatment of major bleeding? Br J Anaesth. 2005; 94: 553-555.

124. Stanworth, S.J., Birchall, J., Doree, C.J., Hyde, C.: Recombinant factor VIIa for the prevention and treatment of bleeding in patients without haemophilia. Cochrane DatabaseSyst Rev. 2007; (2): CD005011.

125. Stein, D.M., Dutton, R.P., Kramer, M.E., Handley, C., Scalea, T.M.: Recombinant factor VIIa: decreasing time to intervention in coagulopathic patients with severe traumatic brain injury. J Trauma. 2008; 64: 620-627.

126. Stein, D.M., Dutton, R.P., Kramer, M.E., Scalea, T.M.: Reversal of coagulopathy in critically ill patients with traumatic brain injury: recombinant factor VIIa is more costeffective than plasma. J Trauma. 2009; 66: 63-72.

127. Stein, D.M., Dutton, R.P., O’Connor, J., Alexander,
M., Scalea, T.M.: Determinants of futility of administration of recombinant factor VIIa in trauma. J Trauma. 2005; 59: 609615 .

128. Steiner, T., Bosel, J.: Options to restrict hematoma expansion after spontaneous intracerebral hemorrhage. Stroke. 2010; 41: 402-409.

129. Steiner, T., Diringer, M.N., Schneider, D., Mayer, S.A., Begtrup, K., Broderick, J., et al.: Dynamics of intraventricular hemorrhage in patients with spontaneous intracerebral hemorrhage: risk factors, clinical impact, and effect of hemostatic therapy with recombinant activated factor VII. Neurosurgery. 2006; 59: 767-773.

130. Strauss, T., Kenet, G., Schushan-Eisen, I., Mazkereth, R., Kuint, J.: Rescue recombinant activated factor VII for neonatal subgaleal hemorrhage. Isr Med Assoc J. 2009; 11: 639-640.

131. Stricker, P.A., Petersen, C., Fiadjoe, J.E., McCloskey, J.J.: Successful treatment of intractable hemorrhage with recombinant factor VIIa during cranial vault reconstruction in an infant. Paediatr Anaesth. 2009; 19: 806-807.

132. Subramaniam, S., Demchuk, A.M., Watson, T., Barber, P.A., Hill, M.D.: Unexpected posthemorrhagic hydrocephalus in patients treated with rFVIIa. Neurology. 2006; 67: 1096.

133. Sucker, C., Sabel, M., Stummer, W., Zotz, R.B., Scharf, R.E., Gerhardt, A.: Recombinant factor VIIa for the prophylaxis of perioperative hemorrhage in a patient with congenital factor XI deficiency undergoing brain tumor neurosurgery. Clin Appl Thromb Hemost. 2008; 14: 472475.

134. Sugg, R.M., Gonzales, N.R., Matherne, D.E., Ribo, M., Shaltoni, H.M., Baraniuk, S., et al.: Myocardial injury in patients with intracerebral hemorrhage treated with recombinant factor VIIa. Neurology. 2006; 67: 1053-105.

135. Sutherland, C.S., Hill, M.D., Kaufmann, A.M., Silvaggio, J.A., Demchuk, A.M., Sutherland, G.R.: Recombinant factor VIIa plus surgery for intracerebral hemorrhage. Can J Neurol Sci. 2008; 35: 567-572.

136. Szabo, T., Ali, S., Camporesi, E.M.: Intraoperative recombinant activated factor VII for emergent epidural hematoma evacuation. Anesth Analg. 2004; 99: 595-597.

137. Tobias, J.D.: Synthetic factor VIIa to treat dilutional coagulopathy during posterior spinal fusion in two children. Anesthesiology. 2002; 96: 1522-1525.

138. Tuhrim, S.: Intracerebral hemorrhage--improving outcome by reducing volume? N Engl J Med. 2008; 358: 2174-2176.

139. Uhrig, L., Blanot, S., Baugnon, T., Orliaguet, G., Carli, P.A., Meyer, P.G.: Use of recombinant activated factor VII in intractable bleeding during pediatric neurosurgical procedures. Pediatr Crit Care Med. 2007; 8: 576-579.

140. Veldman, A., Josef, J., Fischer, D., Volk, W.R.: A prospective pilot study of prophylactic treatment of preterm 
neonates with recombinant activated factor VII during the first 72 hours of life. Pediatr Crit Care Med. 2006; 7: 34-39.

141. Veshchev, I., Elran, H., Salame, K.: Recombinant coagulation factor VIIa for rapid preoperative correction of warfarin-related coagulopathy in patients with acute subdural hematoma. Med Sci Monit. 2002; 8: CS98-100.

142. Vidarsson, B, Onundarson, P.T.: Recombinant factor VIIa for bleeding in refractory thrombocytopenia. Thromb Haemost. 2000; 83: 634-635.

143. Wada, R., Aviv, R.I., Fox, A.J., Sahlas, D.J., Gladstone, D.J., Tomlinson, G., et al.: CT angiography "spot sign" predicts hematoma expansion in acute intracerebral hemorrhage. Stroke. 2007; 38: 1257-1262.

144. White, C.E., Schrank, A.E., Baskin, T.W., Holcomb, J.B.: Effects of recombinant activated factor VII in traumatic nonsurgical intracranial hemorrhage. Curr Surg. 2006; 63: 310-317.

145. Wolzt, M., Levi, M., Sarich, T.C., Bostrom, S.L., Eriksson, U.G., Eriksson-Lepkowska, M., et al.: Effect of recombinant factor VIIa on melagatran-induced inhibition of thrombin generation and platelet activation in healthy volunteers. Thromb Haemost. 2004; 91: 1090-1096.

146. Wong, W.Y., Huang, W.C., Miller, R., McGinty, K., Whisnant, J.K.: Clinical efficacy and recovery levels of recombinant FVIIa (NovoSeven) in the treatment of intracra- nial haemorrhage in severe neonatal FVII deficiency. Haemophilia. 2000; 6: 50-54.

147. Young, W.L.: Anesthesia for endovascular neurosurgery and interventional neuroradiology. Anesthesiol Clin. 2007; 25: 391-412.

148. Yusim, Y., Perel, A., Berkenstadt, H., Attia, M., Knoller, N., Sidi, A.: The use of recombinant factor VIIa (NovoSeven) for treatment of active or impending bleeding in brain injury: broadening the indications. J Clin Anesth. 2006; 18: 545-551.

149. Zaaroor, M., Soustiel, J.F., Brenner, B., Bar-Lavie, Y., Martinowitz, U., Levi, L.: Administration off label of recombinant factor-VIIa (rFVIIa) to patients with blunt or penetrating brain injury without coagulopathy. Acta Neurochir (Wien). 2008; 150: 663-668.

Rama-Maceiras, P.; Ingelmo-Ingelmo, I.; Fàbregas-Julià, N.; Hernández-Palazón, J.: Rol del factor VII recombinante activado en pacientes neuroquirúrgicos $\mathrm{y}$ neurocríticos. Neurocirugía 2011; 22: 209-223.

Correspondencia: Pablo Rama Maceiras. Servicio de Anestesiología y Reanimación. Complejo Hospitalario Universitario A Coruña. C/11 Xubias de Arriba, No 84. 15006 A Coruña.

E-mail: prmaceiras@wanadoo.es 